\title{
The impact of twice-daily consultant ward rounds on the length of stay in two general medical wards
}

\author{
Aftab Ahmad, Tejpal S Purewal, Dushyant Sharma and Philip J Weston
}

\begin{abstract}
Excess average length of stay (ALoS) not only results in an increased cost to hospitals but also increases the risk of hospital-acquired infection and thromboembolism. Various factors suggested to affect ALoS have yet to demonstrate a significant impact in clinical practice. Increased consultant input has been identified as an important factor influencing ALoS. As a result, a radical and innovative consultant job plan, replacing twice-weekly with twice-daily ward rounds (WRs) on a university teaching hospital's two medical wards has been designed. The number of discharges (NoDs) significantly increased $(p<0.01)$, ALoS reduced $(p<0.01)$, whereas, readmission rate and mortality remained unchanged $(p=N S)$ over 12 months following twice-daily WRs compared to two other wards with twice-weekly WRs. This innovative model resulted in almost doubling the NoDs and halving the ALoS. This study suggests that ALoS can be reduced and sustained with a cultural and behavioural shift in consultant working patterns, without affecting readmission rate or inpatient mortality.
\end{abstract}

KEY WORDS: discharges, four-hour target, length of stay, readmission, ward rounds

\section{Introduction}

The Royal Liverpool University Hospital is a large teaching hospital managing unselected acute admissions. Two medical wards are supervised by four consultants, supported by a full medical team. Each ward has 25 beds and patients admitted with unselected acute medical problems are managed and then discharged or transferred to community hospitals. Traditionally, each consultant would provide two ward rounds (WRs) per week to their half of the patients on their respective ward (two consultants based on each ward). An additional senior WR was provided by specialist registrars (SpRs) leaving the junior doctors performing WRs on the other two days on each ward. Patient management by consultants was, therefore, limited to two days a week resulting in patients being reviewed and managed by junior doctors for up to five days, potentially leading to delayed management and discharge.

Aftab Ahmad, consultant physician and clinical director; Tejpal S Purewal, consultant physician; Dushyant Sharma, consultant physician; Philip J Weston, consultant physician

Department of Diabetes/Endocrinology and General Medicine, Royal Liverpool University Hospital

\section{Rationale for intervention}

The NHS has changed over the last 10 years and with the recent financial downturn, hospitals are being challenged to provide high-quality patient care with limited resources. Moreover, clinicians and managers are expected to meet four-hour targets, ${ }^{1}$ where no patient would wait more than four hours in an emergency department (ED), from arrival to admission to a bed in the hospital or discharge, with zero tolerance for hospitalacquired infections (HAIs), ${ }^{2}$ both of which are associated with patient average length of stay (ALoS).$^{3-5}$ High bed occupancy is associated with prolonged admission waits from the ED. ${ }^{6}$ The latter can be reduced by availability of more empty beds, ${ }^{7}$ which requires reducing ALoS that in turn requires increased, yet appropriate, discharges. Prolonged ALoS has been identified as a possible contributory factor to an increased risk of developing HAIs and thromboembolism ${ }^{3-5}$ which have significant health, and potential financial, implications for patients. ${ }^{3}$

Reducing ALoS and increasing safe discharges would increase bed availability and help achieve the four-hour target while reducing the health risks. ${ }^{3-5}$ Two performance reviews and an audit over the last 12 months demonstrated no significant improvement in ALoS and NoDs on the two study wards despite the implementation of various trust initiatives including benchmarking ALoS performance, process mapping, and documenting an estimated date for discharge.

This prompted the exploration of a different strategy with a radical and innovative change in the consultants' job plan and a major cultural and behavioural shift in the traditional working pattern.

\section{Methods}

\section{Intervention}

Based on reports that seniority affects $\mathrm{ALoS}^{9}$ while leadership plays a pivotal role, ${ }^{10}$ a need for frequent senior clinical decision-making and consistency was identified, underpinning the hypothesis that twice-daily consultant WRs would improve discharge planning, leading to an increased number of discharges (NoDs) and reducing ALoS with little impact, or potentially even an improvement, on the 28-day readmission rate and mortality.

Two consultants were timetabled to provide twice-daily WRs on their respective wards on a week-on and week-off (five days a week) basis alternating with the other two consultants who only manage the outpatient clinics during that week. While based on the wards, each consultant provides inpatient cover for 
the week with only one specialty clinic session in the outpatient department. This was a radical shift from the twice-weekly WRs by each consultant prior to the change. The inpatient consultants lead the discharge planning and decision making while providing continuity of care to patients and ensuring discharges are completed with no delays due to lack of decision making. New admissions following discharges on the same day are then reviewed by consultants on the late afternoon WR following the same process. The proposed changes did not increase the working hours or sessions of the consultants or any other staff and did not require extra resources, thus, being cost-neutral. The consultants were providing six to seven direct clinical care sessions per week including two WRs and three to four clinics in the old system and continued to provide six to seven direct clinical sessions with the new job plan with alternating ward rounds and clinics each week, thus, not requiring any clinic cancellations or income loss for the trust.

The changes were planned and implemented after discussions with the consultants and other team members resulting in all consultants agreeing to a team job plan incorporating a radical change in the working pattern. The proposed changes were presented to the trust executive board and, following approval, were communicated to all staff involved with the hospital medical wards and admission units.

\section{Data collection and key measures}

Data were obtained for NoDs, ALoS, readmission rate and bed occupancy, through the hospital clinical information department, from the two studied medical wards as well as from two other wards with a similar caseload. None of the other two medical wards made any such changes and continued to provide traditional twice-weekly consultant WRs with no difference in resources, including staff. The data were collected by the hospital information department on a daily basis and were reported on a weekly and monthly basis to each department:

- ALoS: the difference, in days, between date of discharge and date of admission in the index episode. Discharges on the same day were considered as one-day stays.

- Readmission(\%): any readmission to any specialty within 28 days divided by live discharges.

- Bed occupancy: average number of beds occupied overnight.

- Mortality(\%): total deaths divided by the total discharges.

The change in consultant working pattern commenced on 1 November 2009 and the study aimed to assess its impact on the key measures after the first 12 months of the change compared to the previous 12 months to identify any changes in ALoS and to ensure they were sustainable. Data were obtained for the key measures from November 2008 to October 2010. Key measure comparisons for the same months were also made with the other two medical wards with similar caseloads to signify any improvements on the wards. Statistical analysis was performed on the monthly data using one-way ANOVA with Tukey and Dunnett's C-test applied for multiple comparisons depending on equal or unequal variances, respectively.

\section{Results}

During the study period, the only change and key intervention was that of twice-daily consultant WRs compared to twiceweekly rounds in previous months. Table 1 details the effects of twice-daily consultant WRs on the key measures comparing the data on the wards prior to, and after, the change as well as the comparison with the other medical wards where consultants continued with twice-weekly WRs.

There were no significant differences in NoDs, ALoS, readmission rate, mortality and bed occupancy between the wards and the other two medical wards over the 12 months (November 2008 to October 2009), prior to the key intervention (Table 1).

Following the change in the consultant WRs on 1 November 2009 , the NoDs significantly increased ( $p<0.01)$, ALoS significantly decreased $(\mathrm{p}<0.01)$, bed occupancy decreased $(\mathrm{p}<0.01)$ with no significant change in readmission or mortality rate $(\mathrm{p}=\mathrm{NS})$ over the next 12 months compared to the previous 12 months (Table 1). Interestingly, the NoDs almost doubled to 125 patients/month and ALoS halved to 5.0 days within the first

Table 1. Effects of daily consultant ward rounds on the key measures.

\begin{tabular}{|c|c|c|c|}
\hline \multicolumn{4}{|c|}{ Other medical wards } \\
\hline & Ward 1 & Ward 2 & Our wards \\
\hline \multicolumn{4}{|l|}{ Number of discharges } \\
\hline Nov 2008 to Oct 2009 & $63.0 \pm 11.6$ & $57.5 \pm 14.6$ & $68.6 \pm 13.2^{\mathrm{NS}}$ \\
\hline Nov 2009 to Oct 2010 & $76.1 \pm 10.8$ & $58.5 \pm 18.5$ & $116.1 \pm 14.3^{+*}$ \\
\hline \multicolumn{4}{|l|}{ Average length of stay } \\
\hline Nov 2008 to Oct 2009 & $10.6 \pm 1.5$ & $11.4 \pm 1.7$ & $10.4 \pm 1.5^{\mathrm{NS}}$ \\
\hline Nov 2009 to Oct 2010 & $10.2 \pm 1.3$ & $10.9 \pm 1.9$ & $5.3 \pm 0.8^{+*}$ \\
\hline \multicolumn{4}{|l|}{ Readmission (\%) } \\
\hline Nov 2008 to Oct 2009 & $17.1 \pm 4.5$ & $17.3 \pm 6.5$ & $17.6 \pm 3.7$ \\
\hline Nov 2009 to Oct 2010 & $16.2 \pm 4.3$ & $20.1 \pm 5.0$ & $18.1 \pm 3.2$ \\
\hline \multicolumn{4}{|l|}{ Mortality (\%) } \\
\hline Nov 2008 to Oct 2009 & $4.0 \pm 2.1$ & $5.0 \pm 1.9$ & $3.2 \pm 2.0$ \\
\hline Nov 2009 to Oct 2010 & $3.4 \pm 1.7$ & $5.2 \pm 2.2$ & $3.4 \pm 3.2$ \\
\hline \multicolumn{4}{|l|}{ Bed occupancy (\%) } \\
\hline Nov 2008 to Oct 2009 & $85.4 \pm 3.8$ & $94.2 \pm 3.6$ & $95.3 \pm 2.1$ NS \\
\hline Nov 2009 to Oct 2010 & $95.1 \pm 1.6$ & $91.5 \pm 4.0$ & $87.5 \pm 4.0^{+\S}$ \\
\hline \multicolumn{4}{|c|}{ Values are expressed as means \pm SD. } \\
\hline \multicolumn{4}{|c|}{ Values represent the means per month. } \\
\hline \multicolumn{4}{|c|}{ NS = not significant compared to all wards. } \\
\hline \multicolumn{4}{|c|}{${ }^{*} \mathrm{P}<0.01$, compared to other medical wards. } \\
\hline \multicolumn{4}{|c|}{${ }^{+} \mathrm{P}<0.01$, compared to previous 12 months on our wards. } \\
\hline${ }^{\S} \mathrm{P}<0.05$, compared to oth & r wards. & & \\
\hline
\end{tabular}


month of the change in consultant WRs and this difference was sustained over the 12 months.

None of the other wards showed any significant change in any of the key measures between November 2009 and October 2010 compared to the previous 12 months while continuing with the traditional twice-weekly WRs. Although there was no difference in the key measures between the two sets of wards between November 2008 and October 2009, intervention resulted in a significant increase in NoDs $(\mathrm{p}<0.001)$ and reduction in ALoS $(\mathrm{p}<0.001)$ compared to the other wards over the next 12 months (Table 1).

\section{Discussion}

Excess ALoS results in an increased cost to hospitals and the NHS as well as a potential loss of income for patients and an increased risk of HAIs and thromboembolism. ${ }^{3-5}$ In a US study, a mean saving of $\$ 680$ has been estimated by reducing the ALoS by one day in patients who remain hospitalised after becoming stable without any demonstrable benefits on outcome. ${ }^{11} \mathrm{NHS}$ reforms have incentivised reduction in ALoS and the Department of Health expects trusts to reduce bed days, ${ }^{8}$ thus reducing cost and infection risk. This report demonstrates immediate reduction in ALoS and increased discharges with no change in readmissions or mortality despite the rapid turnover that was maintained for 12 months following increased consultant input.

Reducing ALoS is inevitably linked to improving patient flow through the system. While increasing the number of discharges it is imperative that excellent quality of care remains a top priority. ${ }^{8} \mathrm{ALoS}$ is a widely used indicator of hospital performance and reports have suggested that patients with longer riskadjusted ALoS received poorer quality of care ${ }^{12}$ and are associated with a higher health risk. ${ }^{13}$ In contrast, hospitals managing to reduce ALoS while managing complex patients demonstrated a lower cost of care with good outcomes and no difference in patient satisfaction ${ }^{14}$ and these benefits were achieved with no significant change in mortality and readmission rate.

Identifying factors affecting ALoS is important to devise strategies that can have an impact on reducing inpatient stay. Variables, such as timing of discharge planning, discharge destination, number of patient complications, diagnosis, admitting doctor, type of admission, availability of community services, number of available hospital beds, and so on, have been identified as having an impact on ALoS. ${ }^{15,16}$ Studies reporting on benefits of discharge planning ${ }^{16}$ make it likely that decision-making behaviour of clinicians might be an important determinant of ALoS when appropriate adjustments for case mix have been made. The practice patterns of consultants, junior doctors, nurses and other healthcare professionals are frequently difficult to change and much effort is needed to change clinicians' behaviour towards discharge planning. Although a study on several thousand hospital discharges in the USA suggested that seniority has an impact on ALoS as junior doctors become more experienced, ${ }^{9}$ there is also evidence to suggest that consultants or senior medical staff are not the chief determinants of the amount of time their patients spend in hospital. ${ }^{17-21}$ However, the latter results were based on conventional involvement of senior medical staff with routine WRs and very little structural change to clinicians' behaviour towards consultations.

An audit was performed to assess the impact of trust initiatives to reduce ALoS on two sets of wards, which demonstrated no significant change in ALoS over the last 12 months. Although many factors have been identified as contributors to delayed ALoS, consultants and nursing staff at this teaching hospital reported suboptimal input of senior decision making and follow through of the discharge plan as one of the main reasons for delayed discharges. Although SpRs have the experience to make discharge decisions and junior doctors follow discharge plans, the audit showed decreased NoDs and increased unnecessary investigations on non-consultant WRs. Models or reports involving practice redesigns that used twice-daily consultant WRs to reduce ALoS have not been found despite evidence of the pivotal role of such leadership in reducing ALoS. ${ }^{10}$ The only change in practice at this hospital was that of daily, rather than twice-weekly, consultant involvement in patient management and decision making while maintaining the same working hours, allowing physicians to follow through the progress while maintaining restraints on unnecessary investigations and providing leadership and communication between key staff members. In addition, this allowed daily clinical and leadership training by the consultants for the junior staff, including SpRs. Compared to the other two wards, the ALoS significantly reduced, NoDs increased with no change in readmissions and mortality despite a rapid turnover of patients suggesting safe management when daily WRs were made. Furthermore, these results have been maintained for 12 months demonstrating sustainability of the model. Although such a model has not been tried or reported before in medical wards, this report suggests that it is possible to replicate the model in wards that admit general medical patients with a mix caseload.

Although the national ALoS for various trusts have been reported to be around six to seven days, this includes data from emergency and acute admission departments where ALoS is recorded in hours. Therefore, it is difficult to get a national ALoS for discharging medical specialties and make direct comparisons. To assess the impact of the study's changes, the ALoS before and after the change were compared. The data were also compared with similar case mix wards over the same period where no such changes were made and a significant reduction in ALoS was found. As this trust lies in the middle of the table on combined ALoS, the authors believe that similar wards in other trusts lower in the results table will have similar ALoS and would benefit from the changes suggested in this paper. Even if trusts can reduce ALoS by one or two days using this model, the savings can be considerable. ${ }^{11}$

The observed reduction in bed occupancy may have been a result of the rapid turnover achieved, especially by the late afternoon WRs where patients transferred to the ward through the afternoon were reviewed and discharged within a couple of hours of admission. Reducing ALoS raises questions about 
quality of care and the possible impact of early discharge on readmission rates and worsening outcomes. Studies have reported mixed results on the relationship between shortened ALoS and readmission rate demonstrating either no increase or an increase in the numbers depending on the study ${ }^{14,22}$ with no reports on reduction in readmission rate with longer ALoS as also shown by this study. There seems to be no convincing evidence that decreasing ALoS results in worsening outcomes or mortality. ${ }^{14,23}$ No significant increase in the readmission rate or in-hospital mortality was observed despite the NoDs almost doubling and the ALoS reducing.

Reducing ALoS, by providing daily consultant WRs, presents an excellent opportunity to achieve clinical efficiency by rationalising medical practice, minimising patient risk, providing care continuum to patients, assisting administrators for budget planning and resource allocation, potentially improving patient satisfaction and quality of care ${ }^{24,25}$ while meeting the national targets of reducing waiting times. The already nationally planned increased community services would help further reduce the ALoS quite considerably. ${ }^{15,16}$

\section{Conclusion}

This study has demonstrated that ALoS and NoDs can be significantly improved by changing the working pattern of consultants through improved decision making and discharge planning while providing consistent leadership to the medical team. Daily consultant supervision resulted in safe and appropriate discharges with no increase in readmissions or mortality. More interestingly, the effects of change to daily WRs were observed almost immediately. This study suggests that a cultural and behavioural shift in consultant working patterns can be achieved through innovative job planning, without increasing working hours or using extra resources. This system is sustainable and could possibly be replicated on medical wards with a mix caseload of medical admissions. In the current financial environment, where excellent quality of care has to be provided with limited resources, new and innovative ways of working have to be devised, implemented and shared between trusts.

\section{Competing interests}

All authors have completed the unified competing interest form at www.icmje.org/coi_discaLoSure.pdf and declare that none of the authors or any member of their families have any financial or nonfinancial interests relevant to the submitted manuscript.

\section{Contributors}

AA and PJW conceived and designed the study. AA analysed and interpreted data. All authors drafted and revised the article and approved the final version.

\section{Acknowledgements}

The authors would like to acknowledge the diabetes and management team at the Royal Liverpool University Hospital for their hard work and contribution in making this study a success. They would also like to thank Professor Munir Ahmad, professor of statistics, for his advice and guidance on statistical analysis. Special thanks to Paul Currie, the divisional analyst at the corporate information department, Royal Liverpool Hospital, for providing the data, and to Peter Williams, medical director, Royal Liverpool Hospital, for his continued support.

\section{References}

1 Department of Health. Reforming emergency care. London: DH, 2001:1-9.

2 Department of Health. MRSA objective: recommendations from the National Quality Board. London: DH, 2009.

3 Clarke A. Why are we trying to reduce length of stay? Evaluation of the costs and benefits of reducing time in hospital must start from the objectives that govern change. Qual Health Care 1996;5:172-9.

4 Donowitz L, Wenzel R, Hoyt J. High risk of hospital-acquired infection in the ICU patient. Crit Care Med 1982;10:355.

5 Girou E, Stephan F, Novara A, Safar M, Fagon J. Risk factors and outcome of nosocomial infections: results of a matched case-control study of ICU patients. Am J Resp Crit Care Med 1998;157:1151.

6 Bagust A. Dynamics of bed use in accommodating emergency admissions: stochastic simulation model. BMJ 1999;319:155-8.

7 Cooke M, Wilson S, Halsall J, Roalfe A. Total time in English accident and emergency departments is related to bed occupancy. Emerg Med J 2004:575-6.

8 Department of Health. Discharge from hospital: pathway, process and practice. London: DH, 2003.

9 Rich E, Gifford G, Luxenberg M, Dowd B. The relationship of house staff experience to the cost and quality of inpatient care. JAMA 1990;263:953-7.

10 Pethybridge J. How team working influences discharge planning from hospital: a study of four multi-disciplinary teams in an acute hospital in England. J Interprofessional Care 2004;18:29-41.

11 Fine M, Pratt H, Obrosky D et al. Relation between length of hospital stay and costs of care for patients with community-acquired pneumonia. Am J Med 2000;109:378-85.

12 Thomas J, Guire K, Horvat G. Is patient length of stay related to quality of care? Hosp Health Serv Admin 1997;42:489-507.

13 Zhan C, Miller M. Excess length of stay, charges, and mortality attributable to medical injuries during hospitalization. JAMA 2003;290:1868-74.

14 Davis K, Koch K, Harvey J et al. Effects of hospitalists on cost, outcomes, and patient satisfaction in a rural health system. Am J Med 2000;108:621-6.

15 Lutjens J, Louette R. Determinants of hospital length of stay. J Nurs Admin 1993;23:14-8.

16 Marchette L, Holloman F. Length of stay: significant variables. J Nurs Admin 1986;16:12-9.

17 Griffiths M, Waters W, Acheson E. Variation in hospital stay after inguinal herniorrhaphy. BMJ 1979;1:787.

18 Heasman M. How long in hospital? A study in variation in duration of stay for two common surgical conditions. Lancet 1964;2:539-41.

19 Hulter A. Physicians' outcome predictions for elderly patients. Survival, hospital discharge, and length of stay in a department of internal medicine. Scand J Soc Med 1986;14:127-32.

20 West R, Roberts C. Some observations on the management of appendicitis in Wales. Int J Epidemiol 1974;3:351-7.

21 Westert G. Variation in use of hospital care. Assen: Van Gorcum, 1992.

22 Heeren O, Dixon L, Gavirneni S, Regenold W. The association between decreasing length of stay and readmission rate on a psychogeriatric unit. Psychiatr Serv 2002;53:76-9.

23 McCormick D, Fine M, Coley C et al. Variation in length of hospital stay in patients with community-acquired pneumonia: are shorter stays associated with worse medical outcomes? Am J Med 1999;107: $5-12$. 
24 Beaver C, Zhao Y, McDermid S, Hindle D. Casemix-based funding of Northern Territory public hospitals: adjusting for severity and socioeconomic variations. Health Econom 1998;7:53-61.

25 Xiao J, Douglas D, Lee A, Vemuri S. A Delphi evaluation of the factors influencing length of stay in Australian hospitals. Int J Health Plann Manage 1997;12:207-18.

Address for correspondence: Dr A Ahmad, Department of Diabetes and Endocrinology, Link 7-C, Royal Liverpool

University Teaching Hospital, Prescot Street,

Liverpool L7 8XP.

Email: aftab.ahmad@rlbuht.nhs.uk.

\title{
A year in the Medicinal Garden of the Royal College of Physicians
}

\author{
Dr Henry Oakeley revised edition
}

The RCP medicinal garden contains over 1,000 different plants that are used, or have been used, in medicine during the past two millennia, and ones that commemorate physicians. This illustrated garden journal describes some of the plants as they flower, week by week, through the seasons. There is also a commentary on their uses, past and present, and space for your notes throughout the year.

Published: revised October 2011 ISBN: 9781860164514 Price: $£ 8.00$ UK, $£ 10.00$ overseas (inc post and packing)
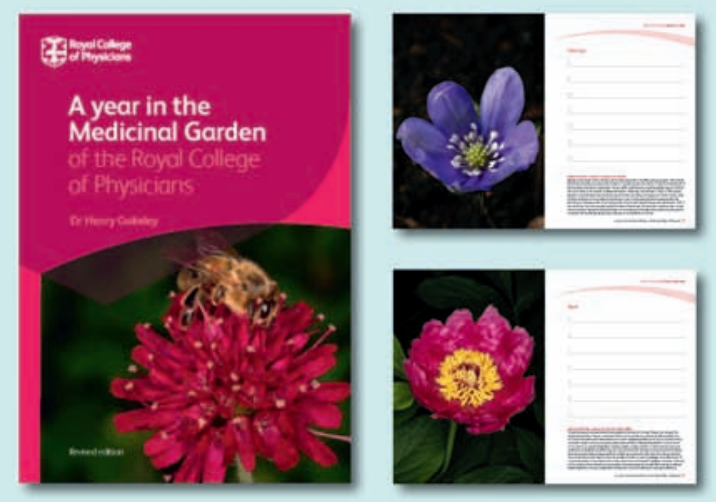